\title{
An end-to-end Fresnel propagation model for SPEED: PIAACMC implementation and performance
}

Mathilde Beaulieu, Patrice Martinez, Lyu Abe, Olivier Guyon, Carole Gouvret, et al.

Mathilde Beaulieu, Patrice Martinez, Lyu Abe, Olivier Guyon, Carole Gouvret, Julien Dejonghe, Oliver Preis, "An end-to-end Fresnel propagation model for SPEED: PIAACMC implementation and performance," Proc. SPIE 10700, Ground-based and Airborne Telescopes VII, 1070030 (6 July 2018); doi: $10.1117 / 12.2311668$

Event: SPIE Astronomical Telescopes + Instrumentation, 2018, Austin, Texas, United States 


\title{
An end-to-end Fresnel propagation model for SPEED : PIAACMC implementation and performance
}

\author{
Mathilde Beaulieu $^{a}$, Patrice Martinez ${ }^{a}$, Lyu Abe ${ }^{a}$, Olivier Guyon ${ }^{b, c, d}$, Carole Gouvret $^{a}$, Julien \\ Dejonghe $^{a}$, Oliver Preis ${ }^{a}$ \\ ${ }^{a}$ Université Côte d'Azur, Observatoire de la Côte d'Azur, CNRS, Laboratoire Lagrange, Bd de \\ l'Observatoire, CS 34229, 06304 Nice cedex 4, France \\ ${ }^{b}$ Astrobiology Center, National Institutes of Natural Sciences, 2-21-1 Osawa, Mitaka, Tokyo, \\ Japan \\ ${ }^{c}$ Steward Observatory, University of Arizona, Tucson, AZ 85721, USA \\ ${ }^{d}$ National Astronomical Observatory of Japan, Subaru Telescope, National Institutes of \\ Natural Sciences, Hilo, HI 96720, USA
}

\begin{abstract}
Future extremely large telescopes, equipped with high-contrast instruments targeting very small Inner Working Angle, will provide the requisite resolution for detecting exoplanets in the habitable zone around M-stars. However, the ELT segmented pupil shape is unfavourable to high-contrast imaging. In this context, the SPEED project aims to develop and test solutions for high contrast with unfriendly apertures. SPEED will combine a PIAACMC coronagraph and two deformable mirrors for the wavefront shaping. In this paper, we describe an end-to-end model of SPEED, including the Fresnel wavefront propagation, the PIAACMC implementation and the dark hole algorithm, and present a statistical analysis of the predicted performance.
\end{abstract}

Keywords: High contrast, PIAACMC, small separation, wavefront control, deformable mirror, Fresnel propagation

\section{INTRODUCTION}

The development of future extremely large telescopes opens a niche for the detection and characterization of faint exoplanets close to their sun. In particular, ESO/ELT will offer the angular resolution that allows the direct imaging of exoplanets, in the habitable zone, around M-star. However, the segmented primary mirror of such telescope $(\sim 800$ segments to achieve diameters of $\sim 40 \mathrm{~m})$ creates gaps between each mirror segments. Those discontinuities, combined with the secondary mirror obstruction and spiders are a major concern for high-contrast imaging that future instrument will have to deal with. The PIAACMC coronagraph (Phase Induced Amplitude Apodization Complex Mask Coronagraph, [1]) has the main advantage to (1) deal with unfriendly pupil shape (gaps between segments, obstruction...) and (2) could provide the adequate contrast and transmission at small separation to detect habitable exoplanets around M-stars [2].

SPEED (Segmented Pupil Experiment for Exoplanet Detection, [3]) is a testbed, started at Lagrange laboratory, Observatoire de la Côte d'Azur, in 2013 in the scientific context of observing planets around M-stars with segmented pupil. The SPEED testbed combines cophasing, coronagraphy and wavefront shaping techniques to achieve high-contrast at small separation $\left(\sim 10^{-6}\right.$ between 1 and a few $\lambda / \mathrm{D}$ in the H-band $)$. The wavefront shaping module will create a high-contrast zone also called dark hole, using the combination of the PIAACMC and two deformable mirrors to correct for both phase and amplitude aberrations over the full field.

Further author information:

M. Beaulieu: E-mail: mathilde.beaulieu@oca.eu, Telephone: 33492076347

Ground-based and Airborne Telescopes VII, edited by Heather K. Marshall,

Jason Spyromilio, Proc. of SPIE Vol. 10700, 107003O · C 2018 SPIE

CCC code: $0277-786 \mathrm{X} / 18 / \$ 18 \cdot$ doi: $10.1117 / 12.2311668$

Proc. of SPIE Vol. 10700 1070030-1 
We have developed simulation tools to study, predict and analyse the SPEED performance. An end-to-end simulation has been developed that includes the Fresnel propagation between each SPEED optic. This paper presents an overview of the SPEED PIAACMC performance and some end-to-end simulation results with this coronagraph.

\section{SPEED OVERVIEW}

The SPEED concept overview is detailed in [4] and [5], and is illustrated in figure 1. A common path (orange line) includes a tip-tilt mirror (TTM) and a segmented deformable mirror (ASM) from IRIS AO* with 163 segments. This segmented mirror is combined with an optical mask (including spiders and obscuration) located near the TTM, to mimic the ELT segmented pupil shape. A dichroïc (DIC) then splits the beam into two bandwidths: the visible path (from 450 to $750 \mathrm{~nm}$ ) for the phasing unit and a NIR (H-band) path for the high-contrast imaging (active optics, coronagraph and NIR camera).

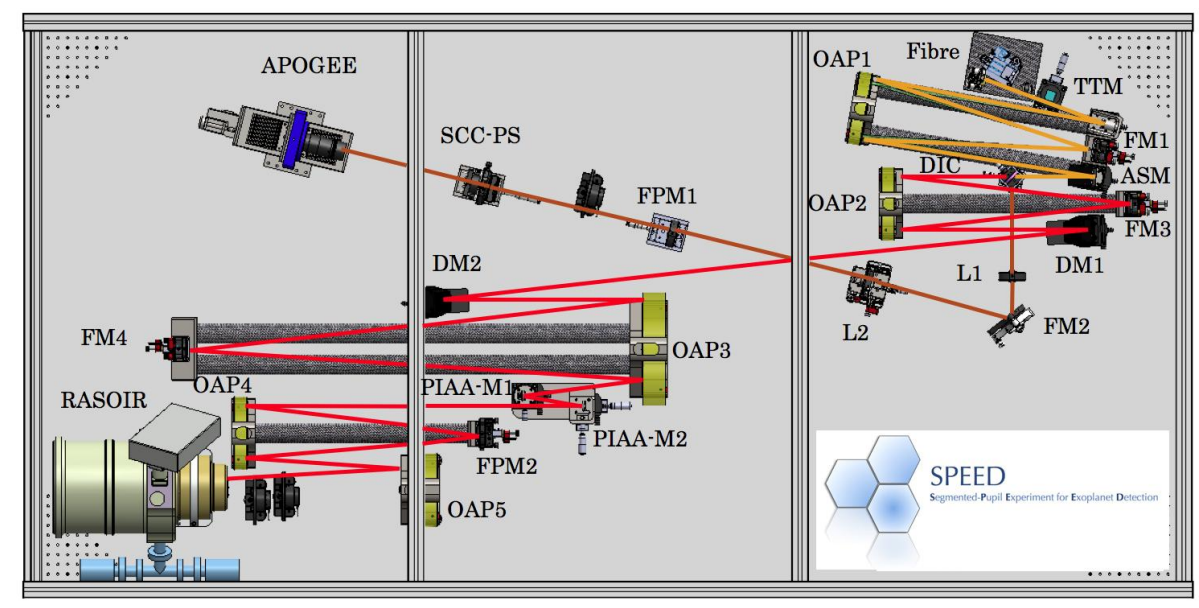

Figure 1. SPEED testbed. The common path (before the dichroï) is shown in orange, the visible path is represented in brown and the NIR path is in red. The acronyms on the figure are : L for lens, OAP for Off-Axis Parabola, ASM for Active Segmented Mirror, DM for Deformable Mirror, FM for Flat Mirror, DIC for dichroïc, SCC-PS for Self-Coherent Camera-Phasing Sensor, FPM for Focal Plane Mask, PIAA-M1 and PIAA-M2 for the two PIAA mirrors for apodization, LS for Lyot Stop, APOGEE for the visible camera and RASOIR for the NIR camera.

The visible path (brown line in figure 1) is dedicated to the cophasing. The proposed technique for SPEED is based on the Self-Coherent Camera (SCC) principle ([6], [7]) and is called the Self-Coherent Camera Phasing Sensor (SCC-PS, [8]). The visible phasing path has been installed and aligned in Lagrange laboratory (see [9] for an updated status of the project).

The NIR path (in red in figure 1), is dedicated to the high-contrast imaging, combining a PIAACMC coronagraph and active optics for the wavefront control. The NIR path is expected to be integrated in laboratory in 2019.

The PIAACMC coronagraph consists in 2 mirrors near a pupil plane that geometrically redirect the light to create an apodized beam. The PIAA concept, proposed in 2004 ([10]) has been validated in laboratory ([11], [12], [13]) and optimized with various pupil shapes and unresolved source [14]. The PIAACMC offers the main advantage of providing high-contrast at small separation while (1) keeping a good overall transmission of the system and (2) dealing with complex and unfriendly pupil shapes (gaps, obscuration...). A description of the SPEED PIAACMC optimization algorithm, tolerancing and manufacturing techniques is detailed in [15]. A brief overview of the PIAACMC design and the theoretical performance simulation are shown in section 3.

The active optics consist in 2 Deformable Mirrors (DMs) from Boston Micromachines ${ }^{\dagger}$, located near the pupil plane, with the role of creating dark holes at the focal plane. Previous numerical simulation had been developed in order to masterize multi-DMs architecture for high-contrast at small separation [16] that used a statistical

\footnotetext{
*http://www.irisao.com

${ }^{\dagger} \mathrm{http}: / /$ www.bostonmicromachines.com
} 
analysis of optics aberrations but assumed a perfect coronagraph. It had contributed to the design definition, by assessing the optimum SPEED optical layout: DM architecture and location. It had been shown that highcontrast at small separation $(1 \lambda / \mathrm{D})$ requiers large setup with large distance between DMs. In this context, the DMs locations for SPEED had been defined, on both sides of the pupil, at $200 \mathrm{~mm}$ and $1500 \mathrm{~mm}$ from the pupil plane [17]. It also had determined the optimum dark hole region that allowed high-contrast around $1 \lambda / \mathrm{D}$, from 0.8 to $4 \lambda /$ D. Section 4 presents the SPEED end-to-end simulation when taking into account the PIAACMC.

\section{PIAACMC DESCRIPTION AND THEORETICAL PERFORMANCE}

The PIAACMC coronagraph has been defined and optimized (see [15] for the optimization code details) with the specific SPEED parameters. The coronagraphic optics are reflective and optimized to provide high-contrast at a IWA of $1.3 \lambda / \mathrm{D}$ at $1.65 \mu \mathrm{m}$ with the SPEED pupil. The SPEED pupil diameter is $7.7 \mathrm{~mm}$ with the shape illustrated in figure 2. As the SPEED region of high contrast is at small separation (1-8 $\lambda / \mathrm{D})$, the PSF distortion

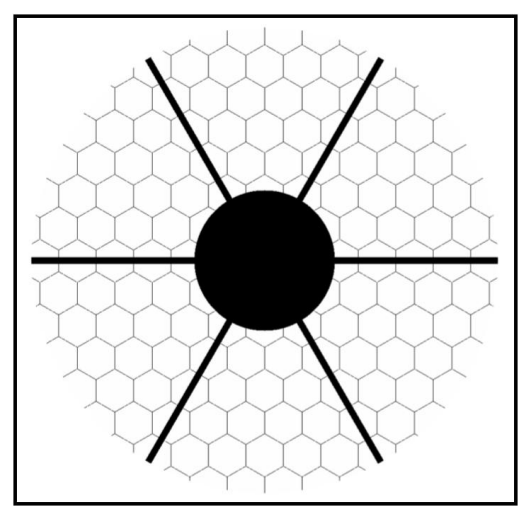

Figure 2. SPEED pupil amplitude.

induced by the mirrors apodization is sufficiently mild to avoid the use of a reverse pair of PIAA mirror, which simplifies the optical design.

An independent cross-checking code has been developed to compute the theoretical (as optimized) performance and define the manufacturing tolerancing of the PIAACMC optics (see [15] for details). We have thus implemented the coronagraphic optics into a simple simulation where we only simulate the Fresnel propagation of the SPEED coronagraphic part, from the PIAA first mirror to the camera (from PIAA-M1 to the camera RASOIR in the optical design presented in figure 1) and without any aberration in the testbed. The Fresnel propagation between each coronagraphic optic is simulated using PROPER [18] that uses the angular spectrum and Fresnel approximation as propagation algorithms.

The two coronagraphic mirrors $\left(P I A A-M_{1}\right.$ and $\left.P I A A-M_{2}\right)$, used to geometrically apodize the beam, are located respectively at the pupil plane and at $150 \mathrm{~mm}$ from the pupil plane and their profiles are centro-symetric (see figure 3).

The PIAACMC Focal Plane Mask (FPM) is made of 498 hexagons, with a size, tip to tip, of $25 \mu \mathrm{m}$ corresponding to a mask outer radius of $4 \lambda / \mathrm{D}$. The FPM sag varies between -800 and $800 \mathrm{~nm}$ as shown in figure 4 . In order to accurately simulate the FPM effect on wavefront, the mask has been defined and optimized with higher resolution than the current simulation resolution (oversampling factor of 8 ). The methodology used to simulate the occulter effect is detailed in [19] and is as following :

(1) The wavefront is propagated up to the FPM plane.

(2) The wavefront is then propagated to a virtual pupil by a Fourier transform and the pupil amplitude $P_{0}$ is recorded. 

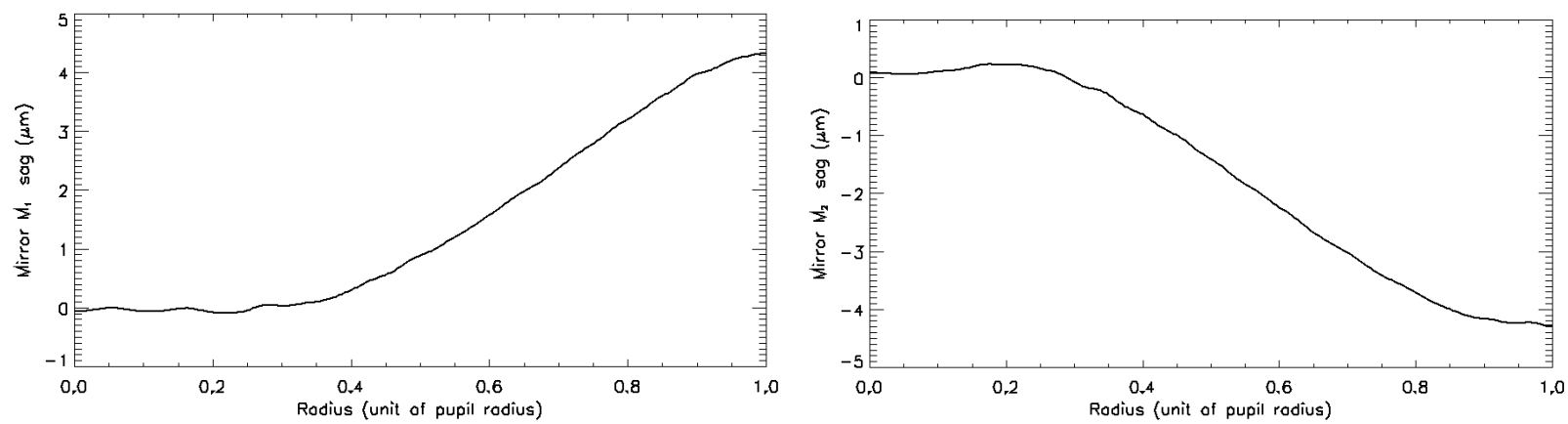

Figure 3. Mirrors sag in $\mu \mathrm{m}: P I A A-M_{1}$ sag on the left and $P I A A-M_{2}$ sag on the right.
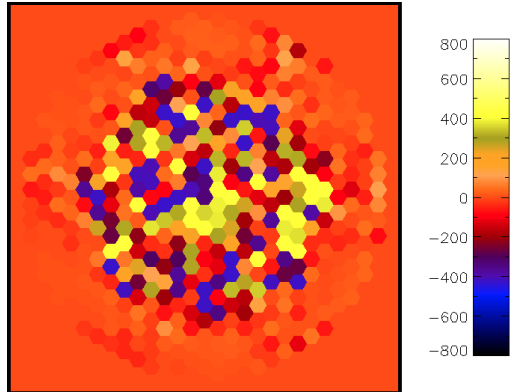

Figure 4. Focal plane mask sag in nm.

(3) A Matrix Fourier Transform (MFT, [20]) allows to compute the wavefront, at a focal plane, with the needed high resolution and within a region where the focal plane mask is active. This oversampled amplitude $A_{\text {no occulter }}$ is recorded.

(4) The oversampled FPM is then applied to the field and recorded $\left(A_{\text {occulter }}\right)$.

(5) The two wavefronts $A_{\text {no occulter }}$ and $A_{\text {occulter }}$ are propagated back to the virtual pupil $\left(P_{n o}\right.$ occulter and $\left.P_{\text {occulter }}\right)$ using a "inverse" MFT.

(6) The effect of the FPM $\left(P_{\text {occulter }}-P_{\text {no occulter }}\right)$ is added to the initial virtual pupil amplitude $P_{0}$.

(7) The wavefront is then Fourier transform and leads to the occulted wavefront definition.

The Lyot Stop is located at $36 \mathrm{~mm}$ from the next pupil plane and has a transmission of $52 \%$. Its amplitude has slightly been modified since the initial optimization simulation, to facilitate the manufacturing. It has been verified that this modified Lyot Stop shape does not significantly degrade the contrast performance. Figure 5 shows the initial (left) and modified (right) Lyot Stop shape.
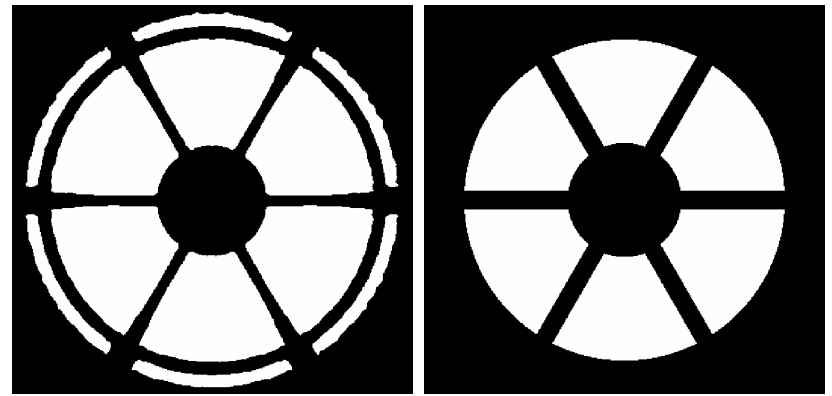

Figure 5. Initital (left) and modified (right) Lyot Stop shapes. 
The contrast intensity has been defined by [21] and corresponds to the PSF halo signal divided by the corresponding signal centered on the unocculted PSF (off-axis PSF). Figure 6 shows the off-axis and coronagraphic PSFs in a logarithmic scale (top of the figure) and the corresponding contrast ratio (bottom). The contrast ratio is better than $10^{-5}$ at IWA $(1.3 \lambda / \mathrm{D})$ and better than $10^{-6}$ at $3 \lambda / \mathrm{D}$.

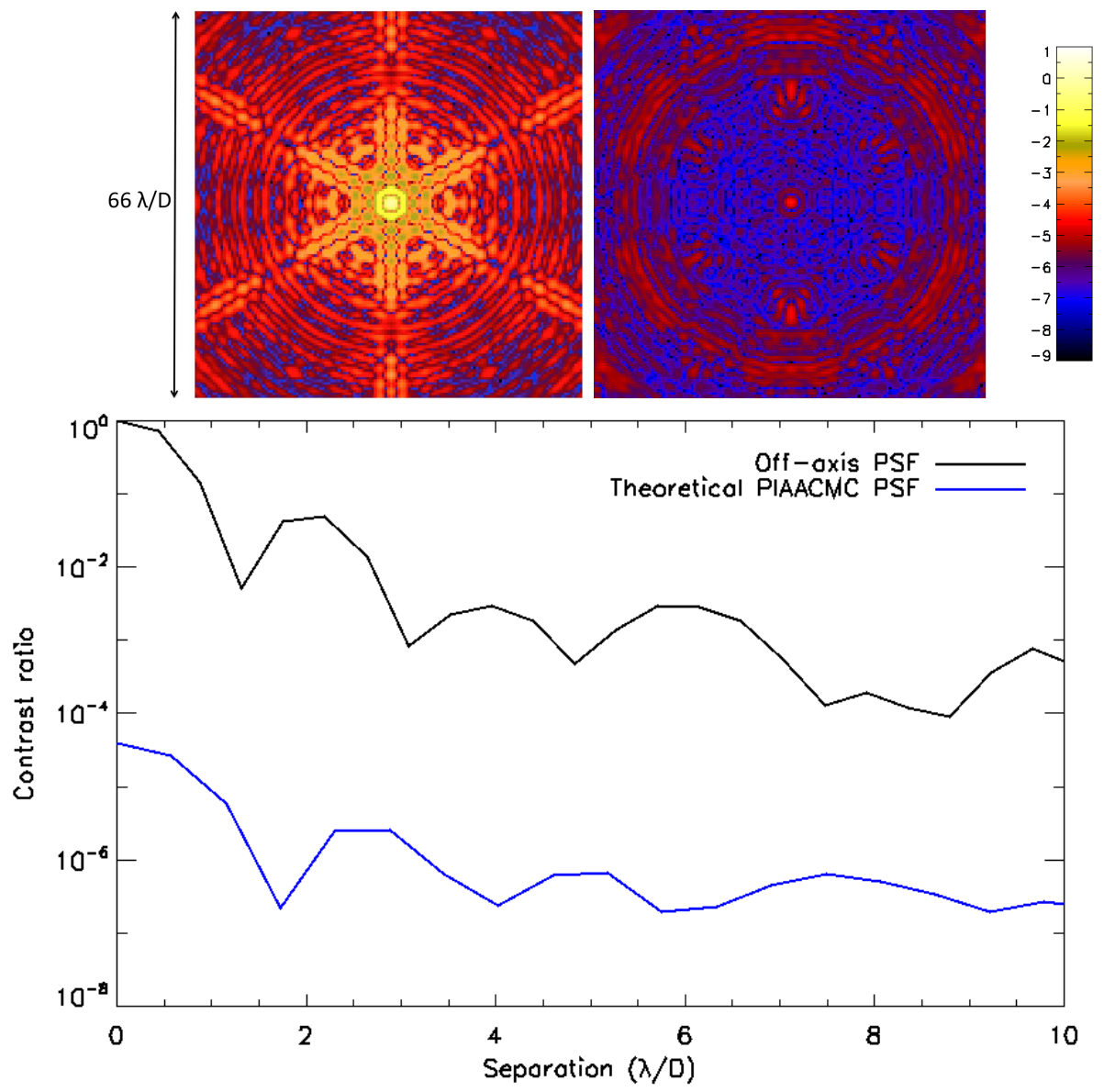

Figure 6. Off-axis and coronagraphic PSFs in a logarithmic scale (top of the figure) and theoretical (as optimized) contrast ratio (bottom).

\section{END-TO-END SIMULATION WITH PIAACMC}

The PIAACMC has been implemented into the SPEED end-to-end simulator. The numerical methodology used for the simulation is detailed in [16] and [22] and is summarized here:

- Fresnel propagation. The Fresnel code for simulation is PROPER. The PROPER routines have been ported to $\mathrm{C}++$, such that the computation can be performed in a parallel system (data centre available at Observatoire de la Côte d'Azur). Paraxial lenses ensure the transition between the image and pupil planes despite the fact that parabolas will be used in SPEED.

- Numerical parameters. The numerical pupil diameter size is 450 pixels for a grid size of 1024 pixels.

- Wavefront sensing. We assume a perfect wavefront sensor that recovers all the complex electric field.

- Wavelength. The simulation is monochromatic at $1.65 \mu \mathrm{m}$.

- Optics phase and amplitude. Each SPEED optic is defined by its amplitude and phase. 


\subsection{Performance without aberration}

In order to assess the impact of the Fresnel propagation on the performance, we have first included the PIAACMC into the overall SPEED design (from the tip-tilt mirror TTM to the camera RASOIR in figure 1) but without adding any optics aberrations and without using the DMs to compute dark region at the final focal plane. Figure 7 illustrates the results obtained when taking into account the SPEED Fresnel propagation (off-axis and on-axis PSFs on top and corresponding contrast ratio at bottom). The SPEED contrast ratio (red curve on the bottom figure) is degraded compared to the theoretical (as optimized) performance (blue curve on the bottom figure), due to the Fresnel propagation at very large distance, as the distance between the first DM and the PIAACMC mirrors (from DM1 to PIAA-M in figure 1) is around $4 \mathrm{~m}$.

In this simulation, optics have a circular shape amplitude, 4 times larger than the pupil diameter, in order to avoid diffraction effect [23]. The SPEED testbed has been defined to respect this rule of thumbs of passive optics $3 / 4$ times larger than the pupil diameter (lens, parabola...). However, the DMs have fixed diameters $(\sim 1.3$ times the pupil diameters) and thus will create diffraction effect. We have then tested the impact of the DM beam clipping on the performance by implementing different DM beam clipping diameters : from 1 to 3 times the pupil diameter, as shown in figure 8 (the DM physical size has been increased for 1 to 3 times the pupil diameter but the DM pitch and actuators number have not been modified). We note that using optics with diameters greater than 3 times the pupil diameter does not impact the coronagraphic performance, which is consistent with the analysis in [23]. The contrast ratio degradation due to the DM size illustrates the need to use the wavefront shaping algorithm to minimize this impact.

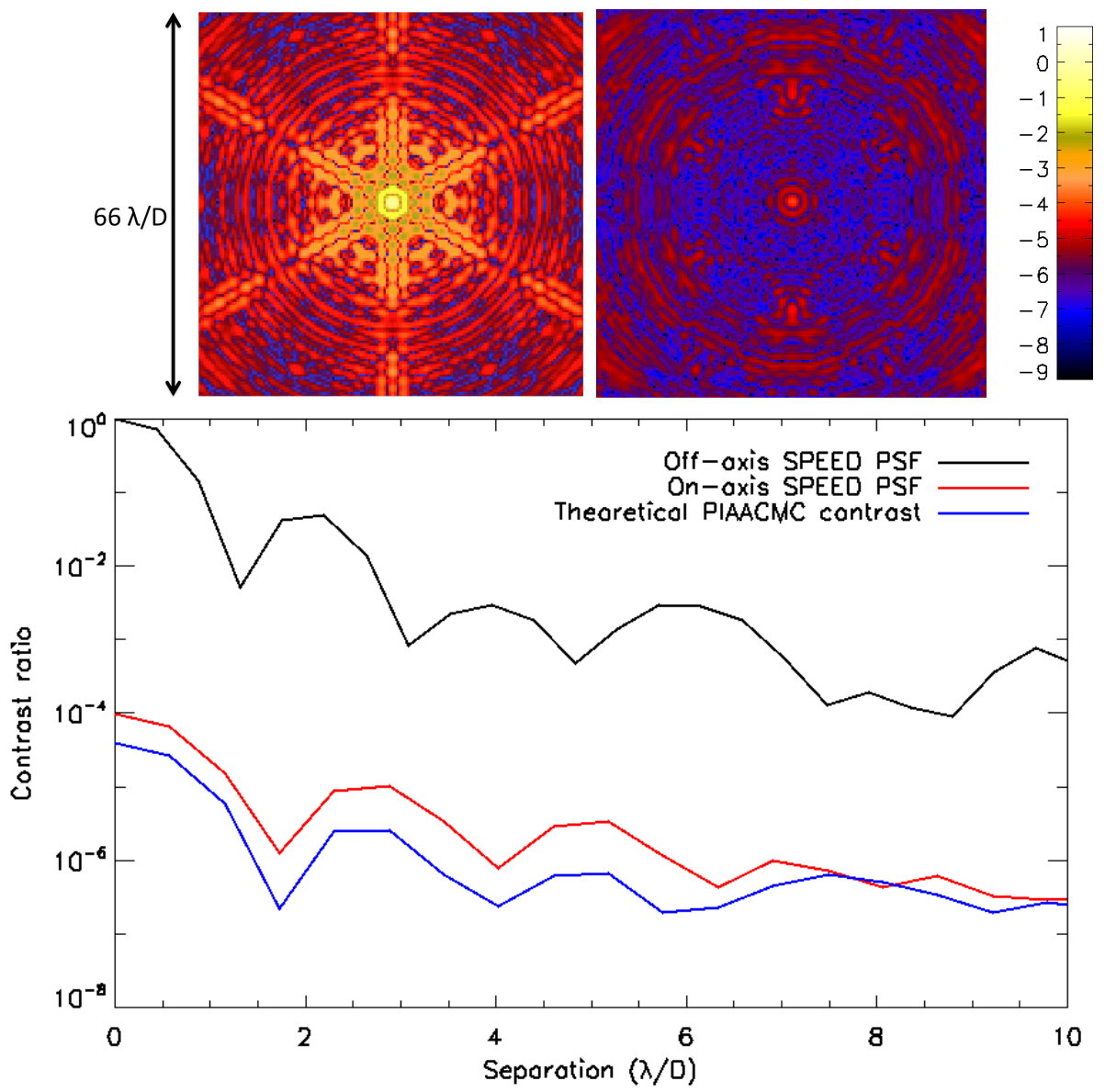

Figure 7. Off-axis and on-axis SPEED PSFs (top left and top right) on logarithmic scale, and corresponding contrast ratio (top). The theoretical PIAACMC contrast corresponds to the as optimized performance. 


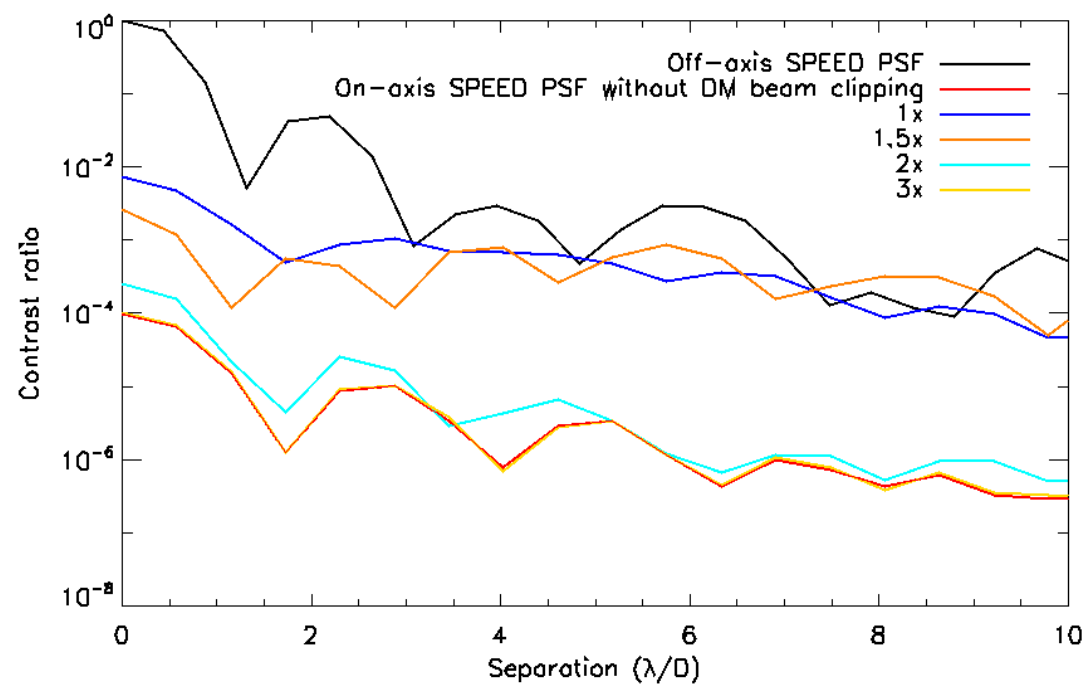

Figure 8. Contrast ratio for off-axis SPEED PSF (black curve), on-axis SPEED PSF without DM beam clipping (red curve) and on-axis SPEED PSFs for DMs beam clipping diameters from 1 to 3 times the pupil diameter (dark blue, orange, light blue and yellow curves).

\subsection{Performance with aberration}

Optics aberrations and dark hole algorithm (with DMs), have then been implemented into the end-to-end simulation. The implementation is as follow:

- Optics phase and amplitude. Each optic has amplitude circular shape set to 4 times the pupil diameter to avoid diffraction effects. The phase aberration pattern (on each optic) is a random realization defined by an amount of $5 \mathrm{~nm}$ rms and a Power Spectrum Density (PSD) power law in $f^{-3}$ (in agreement with the SPEED expected optics qualities). The total amount of aberrations is thus around $20 \mathrm{~nm} \mathrm{rms.} \mathrm{We}$ realized 128 setup phase realizations (each of them consisting of $5 \mathrm{~nm}$ phase aberrations on each SPEED optic) to assess the results repeatability.

- Active optics. The DMs have been simulated with $32 \times 32$ actuators and $300 \mu \mathrm{m}$ pitch. The DM diameter is $10 \mathrm{~mm}$ (1.3 times the pupil diameter).

- Dark hole algorithm. The algorithm is based on a linear approach and minimizes the energy inside the dark hole. This Electric Field Conjugation (EFC) approach is described in [24], [25] and [26] and is defined by first computing the total energy at the image plane with one DM and then generalizing to the case with two DMs. This method uses setup matrix representation to compute the DM coefficients [26]. It consists in finding the DMs coefficients that minimize the energy inside a dark region (dark hole). The interaction matrix, which represents the system response of each DM actuator, is computed by (1) adding a poke to each actuator, (2) propagating the wavefront all along the setup and (3) recording the complex electric field at the focal plane. As this simulation requires large computational time, the code has been transferred to a data center for a gain of time of $\sim 15$ hours.

- Dark hole size. The SPEED field of view is defined from 1 to $8 \lambda /$ D. Previous end-to-end analysis [16] has shown that a small dark hole size is needed to allow high-contrast imaging at small separation. The dark hole size has thus been defined from 0.8 to $4 \lambda / \mathrm{D}$ to asses the performance around $1 \lambda / \mathrm{D}$.

The metric used to determine the performance is the contrast ratio computed inside the dark hole of the PSF divided by the maximum value of the initial PSF without the coronagraphic FPM nor the wavefront shaping. Figure 9 shows the contrast as a function of the phase realization number where each asterisk represents the median contrast ratio computed within a dark hole, defined from 0.8 to $4 \lambda / \mathrm{D}$. Black, red and blue asterisks 


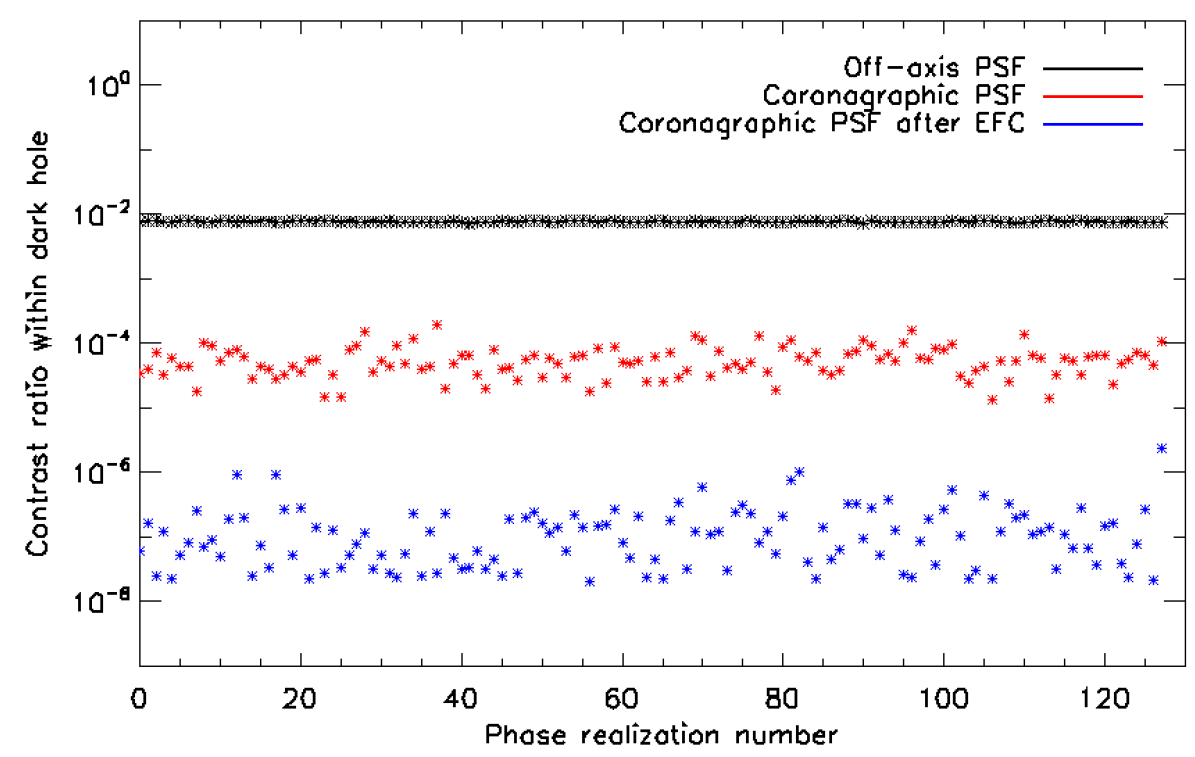

Figure 9. Contrast ratio within the dark hole (from 0.8 to $4 \lambda / \mathrm{D}$ ) as a function of the phase realization number.

represent respectively the contrast ratio for the off-axis PSF (no focal plane mask, no dark hole algorithm), the coronagraphic PSF (without the dark hole algorithm) and the coronagraphic PSF after the dark hole algorithm. The EFC algorithm creates a dark region and allows a gain inside the dark hole of $~ 900$ (mean value of all realizations). The dispersion in realization performance could be explained by (1) the linear approximation, assumed when computing the EFC algorithm, is no more valid when implementing the PIAACMC and the segmented pupil (2) a local minimum is reached during the process.

Figure 10 shows an example of the computed PSFs in a logarithmic scale: off-axis PSF (left), coronagraphic PSF (middle) and coronagraphic PSF after EFC algorithm (right) for the case that represents the median performance.
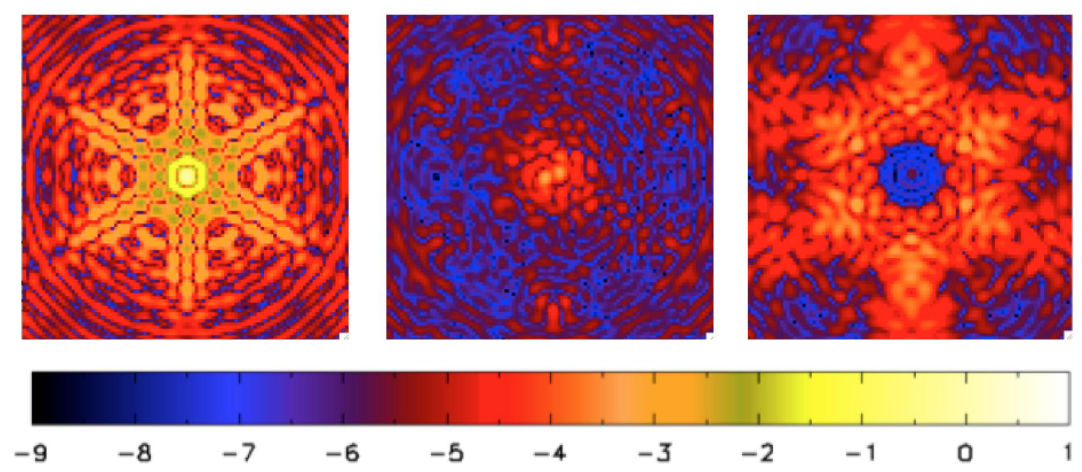

Figure 10. Example of computed PSFs : off-axis PSF (left), coronagraphic PSF (middle) and coronagraphic PSF after EFC algorithm (right) for the case that represents the median performance. The overall size of the display is $44 \mathrm{x} 44 \lambda / \mathrm{D}$.

\section{CONCLUSION}

In this paper, we have presented the SPEED coronagraphic performance. The PIAACMC has been optimized to provide high-contrast at very small separation $\left(10^{-5}\right.$ at $\left.1.3 \lambda / \mathrm{D}\right)$. The amount of expected SPEED aberrations ( $5 \mathrm{~nm}$ rms per optic leading to $20 \mathrm{~nm}$ rms over the full testbed) has been taken into account by generating 128 random phase realizations on each optic for statistical analysis. The value of the contrast ratio median integrated inside the dark hole (from 0.8 to $4 \lambda / \mathrm{D}$ ) goes from $8.10^{-7}$ without aberration to $5.10^{-5}$ with aberration. The 
EFC algorithm has been implemented to generate high-contrast region close to the star (from 0.8 to $4 \lambda / \mathrm{D}$ ). The median contrast ratio achieved is then $10^{-7}$. The real measured optics aberrations will be implemented, in the future, in SPEED to assess the expected performance. A way of optimizing the dark hole algorithm will also been studied (non linearity...).

\section{ACKNOWLEDGEMENT}

The activity outlined in this paper is partially funded by the PACA Region, the French Government, and by the European Union as part of the FEDER programme. The project also benefits from funding support from the Observatoire de la Côte d'Azur, the Lagrange Laboratory, the Nice Sophia-Antipolis University, and the french spatial agency (CNES) through a 2015 R\&T programme. The authors are grateful to the computing centre personal for their support.

\section{REFERENCES}

[1] Guyon, O., Martinache, F., Belikov, R., and Soummer, R., "High Performance PIAA Coronagraphy with Complex Amplitude Focal Plane Masks," ApJS 190, 220-232 (Oct. 2010).

[2] Guyon, O. and Martinache, F., "How Extremely Large Telescopes (ELTs) will Acquire the First Spectra of Rocky Habitable Planets," in [American Astronomical Society Meeting Abstracts \#221], American Astronomical Society Meeting Abstracts 221, 419.05 (Jan. 2013).

[3] Martinez, P., Preis, O., Gouvret, C., Dejongue, J., Daban, J.-B., Spang, A., Martinache, F., Beaulieu, M., Janin-Potiron, P., Abe, L., Fantei-Cujolle, Y., Ottogalli, S., Mattei, D., and Carbillet, M., "The SPEED Project: SPEEDing up Research and Development towards High-contrast Imaging Instruments for the E-ELT," The Messenger 159, 19-22 (Mar. 2015).

[4] Martinez, P., Preis, O., Gouvret, C., Dejonghe, J., Daban, J.-B., Spang, A., Martinache, F., Beaulieu, M., Janin-Potiron, P., Abe, L., Fantei-Caujolle, Y., Mattei, D., and Ottogalli, S., "SPEED: the segmented pupil experiment for exoplanet detection," in [Ground-based and Airborne Telescopes V], Proc. SPIE 9145, 91454E (July 2014).

[5] Martinez, P., Beaulieu, M., Janin-Potiron, P., Preis, O., Gouvret, C., Dejonghe, J., Abe, L., Spang, A., Fantéi-Caujolle, Y., Martinache, F., Belzanne, P., Marcotto, A., and Carbillet, M., "The Segmented Pupil Experiment for Exoplanet Detection: 2. design advances and progress overview," in [Ground-based and Airborne Telescopes VI], Proc. SPIE 9906, 99062V (July 2016).

[6] Baudoz, P., Boccaletti, A., Baudrand, J., and Rouan, D., "The Self-Coherent Camera: a new tool for planet detection," in [IAU Colloq. 200: Direct Imaging of Exoplanets: Sciencea Techniques], Aime, C. and Vakili, F., eds., 553-558 (2006).

[7] Galicher, R., Baudoz, P., Delorme, J. R., Mazoyer, J., Rousset, G., Firminy, J., Boussaha, F., N'Diaye, M., Dohlen, K., and Caillat, A., "High contrast imaging on the THD bench: progress and upgrades," in [Space Telescopes and Instrumentation 2014: Optical, Infrared, and Millimeter Wave], Proc. SPIE 9143, 91435A (Aug. 2014).

[8] Janin-Potiron, P., Martinez, P., Baudoz, P., and Carbillet, M., "The self-coherent camera as a focal plane fine phasing sensor," A $\mathscr{E} A$ 592, A110 (Aug. 2016).

[9] Martinez, P., Janin-Potiron, P., Beaulieu, M., Gouvret, C., Dejonghe, J., Spang, A., Postnikova, M., Baudoz, P., Guyon, O., Preis, O., Abe, L., Marcotto, A., and N'diaye, M., "The Segmented Pupil Experiment for Exoplanet Detection: 3. advances and first light with segments cophasing," Proc. SPIE this conference (2018).

[10] Guyon, O., "Synchronous interferometric speckle subtraction (SISS): a concept to remove speckle noise in adaptive optics imaging and interferometry," in [Advancements in Adaptive Optics], Bonaccini Calia, D., Ellerbroek, B. L., and Ragazzoni, R., eds., Proc. SPIE 5490, 593-601 (Oct. 2004).

[11] Belikov, R., Pluzhnik, E., Connelley, M. S., Witteborn, F. C., Lynch, D. H., Cahoy, K. L., Guyon, O., Greene, T. P., and McKelvey, M. E., "First results on a new PIAA coronagraph testbed at NASA Ames," in [Techniques and Instrumentation for Detection of Exoplanets IV], Proc. SPIE 7440, 74400J (Aug. 2009). 
[12] Guyon, O., Pluzhnik, E., Martinache, F., Totems, J., Tanaka, S., Matsuo, T., Blain, C., and Belikov, R., "High-Contrast Imaging and Wavefront Control with a PIAA Coronagraph: Laboratory System Validation," PASP 122, 71-84 (Jan. 2010).

[13] Kern, B., Belikov, R., Give'On, A., Guyon, O., Kuhnert, A., Levine-West, M. B., McMichael, I. C., Moody, D. C., Niessner, A. F., Pueyo, L., Shaklan, S. B., Traub, W. A., and Trauger, J. T., "Phase-induced amplitude apodization (PIAA) coronagraph testing at the High Contrast Imaging Testbed," in [Techniques and Instrumentation for Detection of Exoplanets IV], Proc. SPIE 7440, 74400H (Aug. 2009).

[14] Guyon, O., Hinz, P. M., Cady, E., Belikov, R., and Martinache, F., "High Performance Lyot and PIAA Coronagraphy for Arbitrarily Shaped Telescope Apertures," ApJ 780, 171 (Jan. 2014).

[15] Martinez, P., Beaulieu, M., Guyon, O., Preis, O., Gouvret, C., Dejonghe, J., Abe, L., Spang, A., and Marcotto, A., "Design, specification and manufacturing of a PIAACMC for the SPEED testbed," Proc. SPIE this conference (2018).

[16] Beaulieu, M., Abe, L., Martinez, P., Baudoz, P., Gouvret, C., and Vakili, F., "High-contrast imaging at small separations: impact of the optical configuration of two deformable mirrors on dark holes," MNRAS 469, 218-230 (July 2017).

[17] Beaulieu, M., Abe, L., Martinez, P., Gouvret, C., Dejonghe, J., Preis, O., and Vakili, F., "SPEED design optimization via Fresnel propagation analysis," in [Ground-based and Airborne Telescopes VI], Proc. SPIE 9906, 99062X (Aug. 2016).

[18] Krist, J. E., "PROPER: an optical propagation library for IDL," in [Optical Modeling and Performance Predictions III], Proc. SPIE 6675, 66750P (Sept. 2007).

[19] Krist, J., Nemati, B., and Mennesson, B., "Numerical modeling of the proposed WFIRST-AFTA coronagraphs and their predicted performances," Journal of Astronomical Telescopes, Instruments, and Systems $\mathbf{2}$, 011003 (Jan. 2016).

[20] Soummer, R., Pueyo, L., Sivaramakrishnan, A., and Vanderbei, R. J., "Fast computation of Lyot-style coronagraph propagation," Optics Express 15, 15935 (2007).

[21] Green, J. J. and Shaklan, S. B., "Optimizing coronagraph designs to minimize their contrast sensitivity to low-order optical aberrations," in [Techniques and Instrumentation for Detection of Exoplanets], Coulter, D. R., ed., Proc. SPIE 5170, 25-37 (Nov. 2003).

[22] Beaulieu, M., Imagerie optique a tres haut contraste : une approche instrumentale optimale, $\mathrm{PhD}$ thesis, France: Universite Cote d'Azur; 2017. Publication Number: tel-01599054 (2017).

[23] Pueyo, L. and Kasdin, N. J., "Polychromatic Compensation of Propagated Aberrations for High-Contrast Imaging," ApJ 666, 609-625 (Sept. 2007).

[24] Give'on, A., Kern, B., Shaklan, S., Moody, D. C., and Pueyo, L., "Broadband wavefront correction algorithm for high-contrast imaging systems," in [Astronomical Adaptive Optics Systems and Applications III], Proc. SPIE 6691, 66910A (Sept. 2007).

[25] Pueyo, L., Kay, J., Kasdin, N. J., Groff, T., Mc Elwain, M., Give'on, A., and Belikov, R., "Optimal Dark Hole Generation via Two Deformable Mirrors with Stroke Minimization," ArXiv e-prints (Nov. 2011).

[26] Groff, T. D., Optimal electric field estimation and control for coronagraphy, PhD thesis, Princeton University (2012). 